\title{
HOMOGENEOUS CONTACT TRANSFORMATIONS
}

\author{
SHIGEO SASAKI
}

(Received July 5, 1962)

Introduction. Let $M^{n}$ be an $n$-dimensional differentiable manifold of class $C^{\infty}$. Take a point $x$ of $M^{n}$ and consider the set $F_{x}$ of all non-zero covectors at $x$. Then, $F_{x}$ with the natural topology is homeomorphic with $F=E^{n}-O$, where $E^{n}$ is a Euclidean space and $O$ is a point of $E^{n}$. We can easily see that the set

$$
{ }^{c} T\left(M^{n}\right)=\bigcup_{x \in \mathscr{M}^{n}} F_{x}
$$

with the natural topology is a fibre bundle with the standard fibre $F$ and the structural group $G L(n, R)$. We shall call this fibre bundle as the cotangent bundle of $M^{n}$.

In this paper, I want so show that cotangent bundles play an important role for the study of homogeneous contact transformations of differentiable manifolds. The classical Laguerre's geometry of $(n-1)$-spheres in $E^{n}$ can be regarded as a geometry of ${ }^{c} T\left(E^{n}\right) \approx E^{n} \times F$ under a certain group of homogeneous contact transformations and the classical Lie's higher $(n-1)$-sphere geometry in $E^{n}$ can be regarded as a geometry of ${ }^{c} T\left(S^{n}\right)$ under a certain group of homogeneous contact transformations, where $S^{n}$ is the $n$-dimensional sphere. Therefore, it is natural to study ${ }^{c} T\left(M^{n}\right)$ in connection with homogeneous contact transformations of $M^{n}$.

On the background of Lie's works L.P. Eisenhart [4] [5] [6] founded the theory of homogeneous contact transformations of a differentiable manifold $M^{n}$ in 1929 and T.Hosokawa [7], K.Yano [2] [9] [10], Y. Mutô [8] [9], T.C.Doyle [3], E.T.Davies [1] [2] and others followed him. From our stand point of view, their theories are local theories of ${ }^{c} T\left(M^{n}\right)$ or tensor calculus of $2 n$ dimensional manifolds under local contact coordinate transformations. It seems to me that their theories can be understood the meaning well by studying the cotangent bundle ${ }^{c} T\left(M^{n}\right)$ globally.

1. Homogeneous contact transformations. Let $M^{n}$ be a differentiable

\footnotetext{
About half of this paper was done when the author was a visiting professor of the National Taiwan University and stayed at the Academia Sinica, Nankang, Formosa from Oct. 1961 to March 1962.
} 
manifold of class $C^{\infty}$ and ${ }^{c} T\left(M^{n}\right)$ be its cotangent bundle. We denote by $\pi$ the natural projection

$$
\pi:{ }^{c} T\left(M^{n}\right) \rightarrow M^{n}
$$

Every point $z$ of ${ }^{c} T\left(M^{n}\right)$ can be expressed as a pair $(x, p)$, where $x=\pi z$ and $p$ is a covector at $x$. We shall call $p$ as the covector of $z$. We sometimes call the pair $(x, p)$ as an element in $M^{n}$.

We take an open covering of $M^{n}$ by coordinate neighborhoods $\left\{U_{\lambda}\right\}(\lambda \in \Lambda)$ and denote local coordinates in $U_{\lambda}$ by $x_{\lambda}^{i}$. If we denote the components of a covector $p$ at any point $x_{(\lambda)}$ in $U_{\lambda}$ with respect to the natural frame $\frac{\partial}{\partial x_{(\lambda)}^{i}}$ at $x_{(\lambda)}$ by $p_{i}^{(\lambda)}$, then the set of all $\left(x_{(\lambda)}^{i}, p_{i}^{(\lambda)}\right)\left(x_{(\lambda)}^{i} \in U_{\lambda}, p_{i}^{(\lambda)} \in F\right)$ constitutes local coordinates in $\pi^{-1}\left(U_{\lambda}\right)$. This mapping

$$
\pi^{-1}\left(U_{\lambda}\right) \rightarrow U_{\lambda} \times F
$$

is a diffeomorphism and its inverse mapping is usually denoted by $\phi_{\lambda}$. So, we have

$$
z=\phi_{\lambda}\left(x_{(\lambda)}, p^{(\lambda)}\right)
$$

We denote the map which transfers $z$ to $p^{(\lambda)}$ by $\rho_{\lambda}$. Then, we have

$$
\rho_{\lambda}: \pi^{-1}\left(U_{\lambda}\right) \rightarrow F,
$$

$\left\{\pi^{-1}\left(U_{\lambda}\right)\right\}(\lambda \in \Lambda)$ is an open covering of ${ }^{c} T\left(M^{n}\right)$ by coordinate neighborhoods.

On every neighborhood $\pi^{-1}\left(U_{\lambda}\right)(\lambda \in \Lambda)$ of ${ }^{c} T\left(M^{n}\right)$ we consider a 1 -form

$$
\eta_{(\lambda)} \equiv p_{i}^{(\lambda)} d x^{i}
$$

As the right hand side is a scalar, it does not depend upon the coordinate transformation. So, the set of all $\eta_{\lambda}(\lambda \in \Lambda)$ constitutes a global 1-form $\eta$ over ${ }^{c} T\left(M^{n}\right)$. We shall call $\eta$ as the homogeneous contact form of $M^{n}$.

$A$ diffeomorphism

$$
f:{ }^{c} T\left(M^{n}\right) \rightarrow{ }^{c} T\left(M^{n}\right)
$$

is said to be a homogeneous contact transformation of $M^{n}$ if and only if $f$ leaves invariant the 1 -form $\eta$, i.e.

$$
f^{*} \eta=\eta
$$

where $f^{*}$ is the dual map induced by $f$ on differential forms over ${ }^{c} T\left(M^{n}\right) . f$ is denoted by

$$
\bar{z}=f(z), \quad z \in{ }^{c} T\left(M^{n}\right)
$$

or by

$$
(\bar{x}, \bar{p})=f(x, p)
$$


where $z=(x, p)$ and $\bar{z}=(\bar{x}, \bar{p})$.

From the definition, we can immediately see that the following theorem is true.

THEOREM 1.1 The totality of homogeneous contact transformations of a differentiable manifold $M^{n}$ forms a group.

EXAMPLE. Suppose $f_{0}$ be a diffeomorphism of $M^{n}$ onto itself. Then $f_{0}$ naturally induces a diffeomorphism $f$ of the cotangent bundle ${ }^{c} T\left(M^{n}\right)$ onto itself. It is easy to see that $f$ is a homogeneous contact transformation. This map $f$ is called to be an extension of the diffeomorphism $f_{0}$ of $M^{n}$.

THEOREM 1.2 A homogeneous contact transformation $f$ of ${ }^{c} T\left(M^{n}\right)$ onto itself is an extension of a diffeomorphism of $M^{n}$ onto itself if and only if $f$ is a fibre preserving map.

The proof is easy.

2. Analytic expressions of homogeneous contact transformations. Let $U$ be a coordinate neighborhood of $M^{n}$ with local coordinates $x^{i}$. We denote components of a covector $p$ at a point $x$ of $U$ with respect to the natural frame by $p_{i}$. For the sake of convenience, we now put

$$
x^{n+i} \equiv x^{i *} \equiv p_{i}, \quad i^{*}=n+i
$$

and consider $x^{\lambda}=\left(x^{i}, x^{n+i}\right)(\lambda, \mu=1, \cdots, 2 n)$ as local coordinates of ${ }^{c} T\left(M^{n}\right)$ in $\pi^{-1}(U)$. Then the contact form $\eta$ over ${ }^{c} T\left(M^{n}\right)$ can be written as

$$
\eta=\eta_{\lambda} d x^{\lambda}
$$

in $\pi^{-1}(U)$, where we have put

$$
\eta_{\lambda}=\left(p_{i}, 0\right) .
$$

$\eta_{\lambda}$ determines a $(2 n-1)$-dimensional vector subspace of the tangent space of ${ }^{c} T\left(M^{n}\right)$ at $(x, p)$ containing the tangent space of the fibre at the point.

Now, we consider the exterior differential $d \eta$ of the contact form $\eta$. In $\pi^{-1}(U)$, it is given by

$$
d \eta=\frac{1}{2} \phi_{\lambda \mu} d x^{\lambda} \wedge d x^{\mu}
$$

where we have put

$$
\phi_{\lambda \mu}=\partial_{\lambda} \eta_{\mu}-\partial_{\mu} \eta_{\lambda}, \partial_{\lambda}=\frac{\partial}{\partial x^{\lambda}} .
$$

We can easily see that $\left(\phi_{\lambda \mu}\right)$ has the following numerical components: 


$$
\left(\phi_{\lambda \mu}\right)=\left(\begin{array}{cc}
0 & -\delta_{i j} \\
\delta_{i j} & 0
\end{array}\right) .
$$

The entity which has components of the form (2.6) for every coordinate neighborhood $\pi^{-1}(U)$ of an open covering of ${ }^{c} T\left(M^{n}\right)$ is a skew-symmetric tensor field of ${ }^{c} T\left(M^{n}\right)$. Geometrically, it gives a null-system in every tangent space of ${ }^{c} T\left(M^{n}\right)$. We shall call it the fundamental null-system of ${ }^{c} T\left(M^{n}\right)$.

We define $\phi^{\lambda \mu}$ for every coordinate neighborhood of the type $\pi^{-1}(U)$ in ${ }^{c} T\left(M^{n}\right)$ by

$$
\phi^{\lambda \mu} \phi_{\mu \nu}=-\delta_{\nu}^{\lambda}
$$

then $\phi^{\lambda \mu}$ 's are components of a skew-symmetric tensor field over ${ }^{c} T\left(M^{n}\right)$ and are given by

$$
\left(\phi^{\lambda \mu}\right)=\left(\begin{array}{cc}
0 & -\delta^{i j} \\
\delta^{i j} & 0
\end{array}\right) .
$$

We define also

$$
\xi^{\lambda}=\phi^{\lambda \mu} \eta_{\mu}
$$

in every $\pi^{-1}(U)$, then $\xi^{\lambda}$ defines a vector field over ${ }^{c} T\left(M^{n}\right)$. Its components in $\pi^{-1}(U)$ are rewritten as

$$
\xi^{\lambda}=\left(0, p_{i}\right)
$$

We can easily see that (2. 9) is equivalent to

$$
\phi_{\lambda \mu} \xi^{\mu}=-\eta_{\lambda} .
$$

Now, suppose $f$ be a homogeneous contact transformation of $M^{n}$. For every pair of coordinate neighborhoods $U$ and $\bar{U}$ with local coordinates $x^{i}$ and $\overline{x^{a}}$ such that $f\left(\pi^{-1}(U)\right) \cap \pi^{-1}(\bar{U})$ is not empty, the restriction map $f \mid \pi^{-1}(U) \cap f^{-1}$ $\left(\pi^{-1}(\bar{U})\right)$ can be expressed analytically by

$$
\overline{x^{\lambda}}=\overline{x^{\lambda}}(x) \text {, }
$$

i.e.

$$
\bar{x}^{a}=\bar{x}^{a}(x, p), \bar{p}_{a}=\bar{p}_{a}(x, p) .
$$

The condition $f^{*} \eta=\eta$ i.e.

$$
\eta_{\alpha} \overline{(x)} \partial_{\lambda} \overline{x^{\alpha}}=\eta_{\lambda}(x)
$$

can be written as

$$
\bar{p}_{a} \partial_{i} \bar{x}^{a}=p_{i}, \quad \bar{p}_{a} \partial^{i} \bar{x}^{a}=0 \quad\left(\partial^{i}=\frac{\partial}{\partial p_{i}}\right) .
$$

From (2.14), we get easily 


$$
\phi_{\alpha \beta} \partial_{\lambda} \bar{x}^{\alpha} \partial_{\mu} \bar{x}^{\beta}=\phi_{\lambda \mu} .
$$

Of course $\phi_{\lambda \mu}$ and $\phi_{\alpha \beta}$ are numerical constants. Contracting $\phi^{\mu \nu} \bar{\partial}_{\gamma} x^{\lambda}\left(\bar{\partial}_{\gamma}=\frac{\partial}{\partial \bar{x}^{\gamma}}\right)$ with (2.16) we get

$$
\phi_{\gamma \delta} \partial_{\mu} \bar{x}^{\delta} \phi^{\mu \nu}=-\bar{\partial}_{\gamma} x^{\nu} .
$$

Contracting $\phi^{\alpha \gamma} \partial_{\nu} \bar{x}^{\beta}$ with the last equation we then get

$$
\phi^{\alpha \beta}=\phi^{\lambda \mu} \partial_{\lambda} \bar{x}^{\alpha} \partial_{\mu} \bar{x}^{\beta} .
$$

From (2.16) we get the following

THEOREM 2.1. The fundamental null-system of the cotangent bundle ${ }^{c} T\left(M^{n}\right)$ is transformed to itself by homogeneous contact transformations.

Now, from the definition we have

$$
\xi^{\alpha}(\bar{x})=\phi^{\alpha \beta} r_{\beta}(\bar{x}) .
$$

Putting (2.14) and (2.18) into the last equation, and making use of (2.9) we get

$$
\xi^{\alpha}(x)=\partial_{\lambda} \bar{x}^{\alpha} \xi^{\lambda}(x) \text {. }
$$

The last equation gives an important theorem:

THEOREM 2.2. The functions $\bar{x}^{a}(x, p)$ and $\bar{p}_{a}(x, p)$ of a homogeneous contact transformation (2.13) are homogoneous of degree 0 and 1 respectively with respect to $p_{i}$.

Proof. By virtue of (2.10), (2.19) is easily seen to be equivalent with

$$
p_{i} \partial^{i} \bar{x}^{a}=0, \quad p_{i} \partial^{i} \overline{p_{a}}=\overline{p_{a}},
$$

which show that $\bar{x}^{a}$ 's and $\bar{p}_{a}$ 's are homogeneous of degree 0 and 1 with respect to $p_{i}$.

Two points $z=(x, p)$ and $z^{\prime}=\left(x, p^{\prime}\right)$ on the same fibre $F_{x}$ of ${ }^{c} T\left(M^{n}\right)$ are said to be equivalent if and only if there exists a constant $\rho \neq 0$ such that

$$
p_{i}^{\prime}=\rho p_{i} \text {. }
$$

We call an equivlaence class in $F_{x}$ as a coray at $x$. The vector $\xi^{\lambda}$ defined in (2.10) is geometrically the tangent vector of the coray. Theorem 2.2 can now be expressed geometrically as follows :

THEOREM 2.3. Every homogeneous contact transformation of $M^{n}$ is a coray preserving diffeomorphism of ${ }^{c} T\left(M^{n}\right)$.

THEOREM 2.4. Let $\bar{x}^{a}$ and $\bar{p}_{a}$ in (2.13) are functions which define a homogeneous contact transformation. Then, we have 


$$
\begin{cases}\partial_{i} \bar{x}^{a}=\bar{\partial}^{a} p_{i}, & \partial^{i} \bar{x}^{a}=-\bar{\partial}^{a} x^{i}, \\ \partial_{i} \bar{p}_{a}=-\bar{\partial}_{a} p_{i}, & \partial^{i} \bar{p}_{a}=\bar{\partial}_{a} x^{i} .\end{cases}
$$

PROOF. By virtue of (2.17), we have

$$
\phi^{\mu \lambda} \partial_{\mu} \bar{x}^{\alpha}=\phi^{\alpha \gamma} \bar{\partial}_{\gamma} x^{\lambda} \text {. }
$$

We can easily see that the last equation is equivalent with (2.22).

Suppose

$$
\begin{aligned}
& X=\left(X^{i}, X^{i^{*}}\right)=\left(X^{i}, P_{i}\right), \\
& Y=\left(Y^{i}, Y^{i^{*}}\right)=\left(Y^{i}, Q_{i}\right)
\end{aligned}
$$

be components of vector fields over ${ }^{c} T\left(M^{n}\right)$. Then, since the numerical components (2. 8) of the tensor field $\phi_{\lambda \mu}$ are kept invariant under homogeneous contact transformations, we see that

$$
\phi_{\lambda \mu} X^{\lambda} Y^{\mu}=-X^{i} Q_{i}+Y^{i} P_{i}
$$

is an invariant under homogeneous contact transformations. Especially, if we take $\xi^{\lambda}$ and $X^{\lambda}$ instead of $X^{\lambda}$ and $Y^{\lambda}$, we see that

$$
\phi_{\lambda \mu} \xi^{\lambda} X^{\mu}=\eta_{\mu} X^{\mu}=p_{i} X^{i}
$$

is an invariant under homogeneous contact transformations.

If $U$ is a function defined over ${ }^{c} T\left(M^{n}\right)$, then

$$
\partial_{\lambda} U=\left(\partial_{i} U, \partial^{i} U\right)
$$

is a covector field over ${ }^{c} T\left(M^{n}\right)$. However,

$$
\phi^{\lambda \mu} \partial_{\mu} U=\left(-\partial^{i} U, \partial_{i} U\right)
$$

is a vector field over ${ }^{c} T\left(M^{n}\right)$.

Let $U, V$ be differentiable functions defined over ${ }^{c} T\left(M^{n}\right)$. We define the socalled Poisson's bracket of $U$ and $V$ by

$$
(U, V)=\phi^{\lambda \mu} \partial_{\lambda} U \partial_{\mu} V=\partial^{i} U \partial_{i} V-\partial_{i} U \partial^{i} V .
$$

Then, $(U, V)$ is also a function defined over ${ }^{c} T\left(M^{n}\right)$. It is evident that if $U, V$ are invariant under homogeneous contact transformation $f$, then $(U, V)$ is also invariant under $f$.

3. Fundamental varieties. Suppose that

$$
f:{ }^{c} T\left(M^{n}\right) \rightarrow{ }^{c} T\left(M^{n}\right)
$$

be a homogeneous contact transformation. Denoting the fibre of ${ }^{c} T\left(M^{n}\right)$ at a point $x$ of $M^{n}$ by $F_{x}$, we put

$$
\begin{aligned}
& S_{x}=\pi \circ f\left(F_{x}\right), \\
& \bar{S}_{x}=\pi \circ f^{-1}\left(F_{x}\right),
\end{aligned}
$$


and call $S_{x}$ and $\overline{S_{x}}$ as the fundamental varieties at $x$ of $f$ and $f^{-1}$ respectively. If $f$ is an extension of a diffeomorphism $f$ of $M^{n}$ onto itself, then it is evident that all fundamental varieties of $f$ and $f^{-1}$ reduce to points.

THEOREM 3.1. If a point $y$ belongs to $S_{x}$. then the point $x$ belongs to $\overline{S_{y}}$. The converse is also true.

PRoOF. $y \in S_{x}$ means that $y \in \pi \circ f\left(F_{x}\right)$ and it is equivalent to $F_{y} \cap f\left(F_{x}\right)$ $\neq \phi$. The last equation can be written also as $f^{-1}\left(F_{y}\right) \cap F_{x} \neq \phi$, and so it is equivalent to $\pi \circ f^{-1}\left(F_{y}\right) \ni x$. Therefore, $x \in \bar{S}_{y}$. The converse can be proved easily by the process inverse to the above.

COROllaRY. (i) If $y \in M^{n}$, then

$$
\overline{S_{y}}=\left\{x \mid y \in S_{x}\right\} .
$$

(ii) If $x \in M^{n}$, then

$$
S_{x}=\left\{y \mid x \in \overline{S_{y}}\right\} .
$$

Now, from $(2.15)_{2}$ we can see that the rank of the matrix $\left(\partial^{i} \bar{x}_{a}\right)$ is smaller than $n-1$. Geometrically, it is nothing but the number of linearly independent tangent vectors at $\bar{x}=\pi \circ f(x, p)$. We shall call it rank of $S_{x}$ at the point $\bar{x}$. So, it is independent upon the choice of coordinate neighborhoods. The variety $S_{x}$ may have singularities in the sense that at some points the rank of $S_{x}$ is less than that of generic points on $S_{x}$. We can see that

$$
\operatorname{dim} S_{x}=\max _{\bar{x} \in S_{x}}\left\{\text { rank of } S_{x} \text { at } \bar{x}\right\} .
$$

For every point $z=(x, p)$ of ${ }^{c} T\left(M^{n}\right)$ we make correspond an integer $r_{f}$ by

$$
r_{f}(z)=\operatorname{rank} \text { of } S_{x} \text { at } \bar{x}=\pi \circ f(z) .
$$

Then, we get an integral valued function $r_{f}$ over ${ }^{c} T\left(M^{n}\right)$ such that

$$
0 \leqq r_{f} \leqq n-1 \text {. }
$$

We call $r_{f}$ as the rank function of the first kind of the homogeneous contact transformation $f$.

THEOREM 3.2. The necessary and sufficient condition that a homogeneous contact transformation $f$ of $M^{n}$ is an extension of a diffeomorphism of $M^{n}$ is that the rank function of the first kind $r_{f}$ of $f$ is identically equal to zero.

ProOF. Necessity. If $f$ is an extension of a diffeomorphism of $M^{n}$, then $S_{x}$ is a point. So $r_{f}$ is equal to zero.

Sufficiency. As $S_{x}$ is arcwise connected, if $r_{f} \equiv 0$, then every $S_{x}$ reduces to a point. So, $f$ is a fibre-preserving diffeomorphism of ${ }^{c} T\left(M^{n}\right)$. Hence, by Theorem 1.3 we can see that $f$ is an extension of a diffeomorphism of $M^{n}$. 
In the next place, we fix a point $z$ in ${ }^{c} T\left(M^{n}\right)$ and take a coordinate neighborhood $U_{\lambda}$ of $\left\{U_{\lambda}\right\}(\lambda \in \Lambda)$ such that $f(z) \in \pi^{-1}\left(U_{\lambda}\right)$. We denote the subset of indices of $\Lambda$ which satisfy the last property by $\Lambda_{z}$. For every $\lambda \in \Lambda_{z}$ we put

$$
\begin{aligned}
& R_{\lambda, \pi(z)}=\rho_{\lambda}\left\{f\left(F_{\pi(z)}\right) \cap \pi^{-1}\left(U_{\lambda}\right)\right\}, \\
& r_{f}^{\prime}\left(z, U_{\lambda}\right)=\text { rank of } R_{\lambda, \pi(z)} \text { at } \rho_{\lambda} \circ f(z) .
\end{aligned}
$$

Analytically, if we denote the coordinate neighborhood of the point $z$ by $\pi^{-1}(U)(\pi z \in U)$ and denote $f$ restricted to $\pi^{-1}(U) \cap f_{0}^{-1} \pi^{-1}\left(U_{\lambda}\right)$ by

$$
x_{(\lambda)}^{a}=x_{(\lambda)}^{a}(x, p), p_{a}^{(\lambda)}=p_{a}^{(\lambda)}(x, p),
$$

then we see easily that

$$
r_{f}^{\prime}(z, U)=\operatorname{rank}\left(\partial^{i} \bar{p}_{a}^{(\lambda)}\right) \text { at }(x, p) .
$$

However, contrary to the rank of $S_{x}$ at a point of $S_{x}, r_{f}^{\prime}\left(z, U_{\lambda}\right)$ depends upon the choice of coordinate neighborhoods. So, we define $r_{f}^{\prime}(z)$ by

$$
r_{f}^{\prime}(z)=\max _{\lambda \in \Lambda} r_{f}^{\prime}\left(z, U_{\lambda}\right) \text {. }
$$

If we vary $z$ over ${ }^{c} T\left(M^{n}\right)$, we get again an integral valued function $r_{f}^{\prime}$ over ${ }^{c} T\left(M^{n}\right)$ depending upon $f$ and such that

$$
0 \leqq r_{f}^{\prime} \leqq n
$$

The function $r_{f}^{\prime}$ generally depends upon the open covering $\left\{U_{\lambda}\right\}$ of $M^{n}$. However, if we consider a covering which contains all possible fine neighborhoods and all possible coordinate systems in them, then $r_{f}^{\prime}$ is a well defined function over ${ }^{c} T\left(M^{n}\right)$ by the homogeneous contact transformation $f$. We shall call $r_{f}^{\prime}$ the rank function of the second kind of the homogeneous contact transformation $f$.

THEOREM. 3.3 At every point $z$ of ${ }^{c} T\left(M^{n}\right)$ and for every homogeneous contact transformation $f$ of $M^{n}$, we have

$$
r_{f}(z)+r_{f}^{\prime}(z) \geqq n \text {. }
$$

Proof. We denote the tangent space of ${ }^{c} T\left(M^{n}\right)$ at $f(z)$ by $T_{f(z)}$ and $U_{\lambda}$ be a coordinate neighborhood such that $\pi \circ f(z) \in U_{\lambda}$. Then, the maximal subspace $V$ (vertical space) of $T_{f(z)}$ such that every vector of $V$ is mapped to zero by $\pi$ and the maximal subspace $H_{\lambda}$ of $T_{f(z)}$ such that every vector of $H_{\lambda}$ is mapped to zero by $\rho_{\lambda}$ are disjoint and complementary.

Now, the dimension of $f\left(F_{\pi(z)}\right)$ at $f(z)$ is $n$. If $\operatorname{dim} S_{\pi \circ f(z)}$ is $n-s$ at $\pi \circ f(z)$, then the $s$-dimensional subspace of $T_{f(z)}$ which is spanned by $s$ independent vectors of $T_{f(z)}$ such that each of them is mapped to zero by $\pi$ is mapped to $s$-dimensional subspace of the standard fibre $F$ by $\rho_{\lambda}$. Therefore, the dimension of $R_{\lambda_{1} \pi(z)}$ is at least $s$. Hence, we get (3.11).

As we have proved it in Theorem 3.1, if a point $\bar{x}$ belongs to $S_{x}$, then 
the point $x$ belong to $\bar{S}_{\bar{x}}$. Any pair of points $x$ and $\bar{x}$ which are in such relation is called to be in the relation $S$.

Now, suppose $\bar{M}^{n}$ is a copy of $M^{n}$ and we consider the point $\bar{x}$ as a point in $\bar{M}^{n}$. Then, the set $\Sigma$ of all pairs $(x, \bar{x})$ in the relation $S$ can be regarded as a submanifold of $M^{n} \times \bar{M}^{n}$. It may have some singularities. It is clear that

$$
\Sigma=\bigcup_{x \in \mathbb{M}^{n}}\left\{x, S_{x}\right\}=\bigcup_{\bar{x} \in \mathbb{M}^{n}}\left\{\bar{S}_{\bar{x}}, \bar{x}\right\}
$$

When $\left(x_{0}, \bar{x}_{0}\right)$ belongs to $\Sigma$, we take coordinate neighborhoods $U$ of $x_{0}$ and $\bar{U}$ of $\bar{x}_{0}$ and we express the homogeneous contact tranrformation $f$ restricted to $\pi^{-1}(U) \cap f^{-1}\left(\pi^{-1}(\bar{U})\right)$ by

$$
\bar{x}^{a}=\bar{x}^{a}(x, p), \bar{p}_{a}=\bar{p}_{a}(x, p) .
$$

If $S_{x}(x \in U)$ has a constant rank for every $(x, \bar{x})$ over a subdomain of $\Sigma$ which contains $\left(x_{0}, p_{0}\right)$, then equations of $\Sigma$ in a neighborhood of $\left(x_{0}, \bar{x}_{0}\right)$ are given by

$$
F_{\sigma}\left(x^{1}, \cdots, x^{n} ; \bar{x}^{1}, \cdots, \bar{x}^{n}\right)=0 \quad(\sigma=1, \cdots, s)
$$

which are obtained by eliminating $p_{\alpha}$ 's from the first equation of (3.13). It is evident that the number $s$ is equal to $n$ minus the constant rank of $S_{x}, x \in U$.

THEOREM 3.4. If $(x, \bar{x})$ be a pair of points in the relation $S$, then the rank of $S_{x}$ at $\bar{x}$ is equal to the rank of $\bar{S}_{\bar{x}}$ at $x$.

PROOF. We consider the rank of $\Sigma$ at the point $(x, \bar{x})$. Then, we can easily see that it is equal to (rank of $S_{x}$ at $\left.\bar{x}\right)+n$ and (rank of $\bar{S}_{\bar{x}}$ at $\left.x\right)+n$. So we have

$$
\operatorname{rank} \text { of } S_{x} \text { at } \bar{x}=\operatorname{rank} \text { of } \bar{S}_{\bar{x}} \text { at } x \text {. }
$$

4. Integral submanifolds. The homogeneous contact form $\eta$ of $M^{n}$ determines an $(2 n-1)$-dimensional distribution defined by

$$
\eta=0 \text {. }
$$

We shall call it the fundamental distribution of the cotangent bundle ${ }^{c} T\left(M^{n}\right)$.

Suppose $N$ be a differentiable submanifold of ${ }^{c} T\left(M^{n}\right)$ and

$$
\iota: N \rightarrow{ }^{c} T\left(M^{n}\right)
$$

be the injection map. If

$$
\iota^{*} \eta=0
$$

then $N$ is said to be an integral submanifold of the fundamental distribution of an integral submanifold for brevity. 
THEOREM 4.1. A submanifold $N$ of ${ }^{c} T\left(M^{n}\right)$ is an integral submanifold if and only if every point $z_{0}$ of $N$ has the property that its covector $p_{0}$ is tangent to $\pi N$ at $x_{0}=\pi z_{0}$.

Proof. Take a coordinate neighborhood $U$ of $M^{n}$ with coordinates $x^{i}$ so that $\pi^{-1}(U)$ contains the point $z_{0}$ of $N$. We also take a coordinate neighborhood $V$ of $N$ with coordinates $\left(u^{1}, \cdots, u^{r}\right), r=\operatorname{dim} N$, so that $V$ contains the point $z_{0}$. Then, a sufficiently small neighborhood of $z_{0}$ with respect to $N$ can be expessed analytically as

$$
x^{i}=x^{i}\left(u^{1}, \cdots, u^{r}\right), p_{i}=p_{i}\left(u^{1}, \cdots, u^{r}\right) .
$$

The condition (4.2) can now be written as

$$
p_{i}(u) \frac{\partial x^{i}}{\partial u^{\lambda}}=0 \quad(\lambda=1, \cdots, r) .
$$

As (4. 3) is the equation of $\pi N$ in the neighborhood of $x_{0}$, the last equation shows that $p_{0}$ is tangent to $\pi N$ at $x_{0}$.

Conversely, if $p_{0}$ is tangent to $\pi N$ at $x_{0}$ for every point $z_{0}$ of $N$, then we have (4. 4) identically. So, we see that (4.2) is true. Hence, $N$ is an integral submanifold.

EXAMPLE 1. Every point of ${ }^{c} T\left(M^{n}\right)$ is a zero dimensional integral submanifold.

EXAMPLE 2. Every fibre $F_{x}\left(x \in M^{n}\right)$ of ${ }^{c} T\left(M^{n}\right)$ is an $n$-dimensional integral submanifold.

THEOREM 4.2. Let $N$ be an integral submanifold in ${ }^{c} T\left(M^{n}\right)$. If $f$ is a homogeneous contact transformation of $M^{n}$, then $f N$ is also an integral submanifold in ${ }^{c} T\left(M^{n}\right)$.

Proof. We denote the injection map of $N$ into ${ }^{c} T\left(M^{n}\right)$ by $\iota$. Then, the injection map of $f N$ into ${ }^{c} T\left(M^{n}\right)$ is given by $f \circ \iota$. As

$$
\begin{aligned}
(f \circ \iota)^{*} \eta & =\iota^{*} \circ f^{*} \eta \\
& =\iota^{*} \eta=0,
\end{aligned}
$$

we can see that $f N$ is an integral submanifold.

COROLLARY. If $f$ is a homogeneous contact transformation of $M^{n}$, then the images $f\left(F_{x}\right)$ and $f^{-1}\left(F_{x}\right)$ of a fibre $F_{x}$ at $x \in M^{n}$ are integral submanifolds.

An important consequence of the last corollary is the following

THEOREM 4.3. Let $z$ be a point of ${ }^{c} T\left(M^{n}\right)$ and $\bar{z}$ be the image of it under a homogeneous contact transformation $f$. Then the covector $\bar{p}$ of $z$ is 
tangent to $S_{x}$ at $\bar{x}=\pi \bar{z}$ and the covector $p$ of $z$ is tangent to $\overline{S_{\bar{x}}}$ at $x=\pi z$.

PROOF. As a point of ${ }^{c} T\left(M^{n}\right), z=(x, p)$ belongs to $F_{x}$ and so $\bar{z}=(\bar{x}, \bar{p})$ belongs to $f\left(F_{x}\right)$. However, by virtue of the last Corollary, $f\left(F_{x}\right)$ is an integral submanifold in ${ }^{c} T\left(M^{n}\right)$. Therefore, $\bar{p}$ is tangent to $\pi \circ f\left(F_{x}\right)=S_{x}$.

In the same way, $p$ is tangent to $\pi \circ f^{-1}\left(F_{\bar{x}}\right)=\overline{S_{\bar{x}}}$.

THEOREM 4.4. The dimensions of integral submanifolds of the homogeneous contact form $\eta$ of a differentiable manifold $M^{n}$ can not be greater than $n$.

PROOF. Let $N$ be an integral submanifold and $z \in N$. We denote the rank of $\pi N$ at $\pi z$ by $r$. Then the dimension of the set of covectors which are tangent to $\pi N$ at $z$ is clearly $n-r$. Hence, the dimension of $N$ is at most $r+(n-r)$, which is to be proved.

Now, we define $F_{x, \bar{x}}$ by

$$
F_{x, \bar{x}}=\left\{z \mid z \in F_{x}, f(z) \in F_{\bar{x}}\right\} .
$$

Then, we get the following

THEOREM 4.5. Suppose $f$ is a homogeneous contact transformation. Then, in order that a covector $\bar{p}$ at a point $\bar{x}$ be tangent to $S_{x}=\pi \circ f\left(F_{x}\right)$, it is necessary and sufficient that $\bar{z}=(\bar{x}, \bar{p})$ is the image under $f$ of an element of $F_{x, \bar{x}}$.

ProOF. Necessity. If $\bar{p}$ is tangent to $S_{x}$ at $\bar{x}$, then

$$
\bar{z}=(\bar{x}, \bar{p}) \in F_{\bar{x}} \cap f\left(F_{x}\right) \text {. }
$$

Therefore,

$$
z=(x, p)=f^{-1}(\bar{z}) \in F_{x}
$$

Hence,

$$
z \in F_{x, \bar{x}}
$$

Sufficiency. If $\bar{z}=f(z), z \in F_{x, \bar{x}}$, then $\bar{z} \in f\left(F_{x}\right)$. As $f\left(F_{x}\right)$ is an integral submanifold, $\bar{p}$ is tangent to $S_{x}$ at $\bar{x}$.

Suppose $N^{n-1}$ be an $(n-1)$-dimensional orientable submanifold of $M^{n}$. At every point of $N^{n-1}$ we take a unit tangent covector of $N^{n-1}$ with respect to an arbitrary but fixed Riemannian metric of $M^{n}$. Then, all such unit covectors constitute a differentiable field over $N^{n-1}$ and the set of elements $\left(x, p_{x}\right)$, where $x \in N^{n-1}$ and $p_{x}$ is the unit tangent covector at $x$ defined above, determines an $(n-1)$-dimensional submanifold in ${ }^{c} T\left(M^{n}\right)$. We shall call it the lift of $N^{n-1}$ and denote it by $l N^{n-1} . l$ may be regarded as a diffeomorphism 


$$
l: N^{n-1} \rightarrow l N^{n-1}
$$

whose inverse is the restriction map $\pi \mid l N^{n-1}$. It is evident that $l N^{n-1}$ is an integral submanifold of $M^{n}$.

Now, suppose that $f$ is a homogeneous contact transformation. Then, $f \circ l N^{n-1}$ is also an $(n-1)$-dimensional integral submanifold. However,

$$
\bar{N}=\pi \circ f \circ l N^{n-1}
$$

is not necessarily $(n-1)$-dimensional. $\bar{N}$ is said to be the image of $N^{n-1}$ under $f$.

EXAMPLE. Consider a dilatation $f$ in Euclidean space $E^{n}$. Then, for any point $y \in E^{n}, \overline{S_{y}}=\pi \circ f^{-1}\left(F_{y}\right)$ is an $(n-1)$-dimensional sphere in $E^{n}$. If we take $\bar{S}_{y}$ with unit tangent covectors as $N^{n-1}$

$$
\begin{aligned}
\bar{N} & =\pi \circ f \circ l \circ N^{n-1} \\
& =\pi \circ f \circ l \circ \pi \circ f^{-1} F_{y}=y .
\end{aligned}
$$

So, $\bar{N}$ is a point. Therefore, $\bar{N}$ is 0 -dimensional.

Now, if we put

$$
\left(\bar{x}, \bar{p}_{\bar{x}}\right)=f\left(x, p_{x}\right)
$$

then $\bar{p}_{\bar{x}}$ is tangent to $\bar{N}$ at $\bar{x}$, as $f \circ l N^{n-1}$ is an integral submanifold in ${ }^{c} T\left(M^{n}\right)$.

Suppose $N_{1}{ }^{n-1}, N_{2}{ }^{n-1}$ be two $(n-1)$-dimensional orientable submanifolds in $M^{n}$ such that they are tangent at a point $x_{0}$. Then, we may construct unit covector fields over $N_{1}^{n-1}$ and $N_{2}^{n-1}$ so that they have $\left(x_{0}, p_{x_{0}}\right)$ in common. If we construct $l N_{1}{ }^{n-1}, l N_{2}{ }^{n-1}$, then they have a point in common and so $f \circ l N_{1}{ }^{n-1}$, $f \circ l N_{2}{ }^{n-1}$ have a point in common too. Therefore,

$$
\bar{N}_{1}=\pi \circ f \circ l N_{1}{ }^{n-1}, \bar{N}_{2}=\pi \circ f \circ l N_{2}{ }^{n-1}
$$

have a common tangent covector at the point $\bar{x}_{0}=\pi f\left(x_{0}, p_{0}\right)$. Hence, we get the following

THEOREM 4.6. Let $N_{1}^{n-1}$ and $N_{2}{ }^{n-1}$ be two $(n-1)$-dimensional orientable submanifolds in $M^{n}$ such that they are tangent at a point. Then, the images of $N_{1}{ }^{n-1}$ and $N_{2}{ }^{n-1}$ under a homogeneous contact transformation have a tangent covector in common.

If the images $\bar{N}_{1}$ and $\bar{N}_{2}$ are both $(n-1)$-dimensional at $\pi \circ f\left(x_{0}, p_{0}\right)$, then they are tangent to each other in the proper sense and this is the reason why our diffeomorphism of ${ }^{c} T\left(M^{n}\right)$ is called to be a (homogeneous) contact transformation.

In the above argument, the fact that $N_{1}{ }^{n-1}$ and $N_{2}{ }^{n-1}$ are submanifolds of $M^{n}$ in the proper sense is not essential. To get the same result, it is essential that $l N_{1}{ }^{n-1}$ and $l N_{2}{ }^{n-1}$ have only a point in common. So, instead of $l N_{1}{ }^{n-1}$ and 
$l N_{2}{ }^{n-1}$ we may take $l N_{1}{ }^{n-1}$ and $F^{*} x_{0}$ as they have just a point in common, where $F^{*} x_{0}$ is the submanifold of $\mathrm{F}_{x_{0}}$ whose points consist of units covectors. This leads us to the following

THEOREM 4.7. Let $N^{n-1}$ be an $(n-1)$-dimensional orientable submanifold in $M^{n}$. If $x_{0} \in N^{n-1}$, then the image of $N^{n-1}$ under a homogeneous contact transformation has a tangent covector in common with $S_{x_{0}}$.

Therefore, in the favourable case when the image $\bar{N}$ of $N^{n-1}$ and $S_{x}\left(x \in N^{n-1}\right)$ are all $(n-1)$-dimensional, $\bar{N}$ is an envelope of $S_{x}$ 's $x \in N^{n-1}$.

\section{Lie algebra of infinitesimal homogeneous contact transformations.}

A vector field $X^{\lambda}=\left(X^{i}, P_{i}\right)$ over ${ }^{c} T\left(M^{n}\right)$ is said to be an infinitesimal homogeneous contact transformation if it satisfies

$$
\mathcal{L}_{0}(X) \eta_{\lambda}=0,
$$

where $\mathcal{L}_{\mathcal{O}}(X)$ means the operator of Lie derivation with respect to the vector field $X$.

THEOREM 5.1. The set $L$ of all infinitesimal homogeneous contact transformations of $M^{n}$ constitutes a Lie algebra with respect to the usual bracket operation.

ProOF. By virtue of the property of the Lie derivative

$$
\mathcal{L}_{0}(X) \mathcal{L}_{0}(Y)-\mathcal{L}_{0}(Y) \mathcal{L}_{0}(X)=\mathcal{L}_{(}([X, Y]),
$$

it is clear that if $X$ and $Y$ are infinitesimal homogeneous contact transformation, then $[X, Y]$ is also an infinitesinal homogeneous contact transformation. Therefore, we can easily see that our theorem is true.

The equation (5. 1) is equivalent to

$$
p_{i} \partial_{j} X^{i}=-P_{j}, p_{i} \partial^{j} X^{i}=0 .
$$

If we put

$$
U=\eta_{\lambda} X^{\lambda}=p_{i} X^{i}
$$

then we have

$$
\begin{aligned}
& \partial_{j} U=p_{i} \partial_{j} X^{i}=-P_{j}, \\
& \partial^{j} U=X^{j}+p_{i} \partial^{j} X^{i}=X^{j}
\end{aligned}
$$

and

$$
p_{j} \partial^{j} U=p_{j} X^{j}=U
$$

So, $U$ is a coray function of degree 1 over ${ }^{c} T\left(M^{n}\right)$ and $X^{\lambda}$ can be written as 


$$
X^{\lambda}=-\phi^{\lambda \mu} \partial_{\mu} U=\left(\partial^{i} U,-\partial_{i} U\right) \text {. }
$$

Conversely, every vector field over ${ }^{c} T\left(M^{n}\right)$ of the form (5.6), where $U$ is a coray function of degree 1 over ${ }^{c} T\left(M^{n}\right)$ is easily seen to be an infinitesimal homogeneous contact transformation. Hence, we get the

THEOREM 5.2. Every infinitesimal homogeneous contact transformation $X$ of a differentiable manifold $M^{n}$ can be written as (5. 6), where $U$ is a coray function of degree 1 . The converse is also true.

The function $U$ is said to be the characteristic function of the infinitesimal homogeneous contact transformation $X$.

N.B. We can easily verify that (5.3) is equivalent to any one of the three equations

$$
\mathcal{L}(X) \xi^{\lambda}=0,[\xi, X]=0, \mathcal{L}_{(}(\xi) X^{\lambda}=0
$$

and

$$
\mathcal{L}(X) \phi^{\lambda_{\mu}}=0 .
$$

THEOREM 5.3. Let $X^{\lambda}=\left(X^{i}, P_{i}\right), Y^{\lambda}=\left(Y^{i}, Q_{i}\right)$ be infinitetimal homogeneous contact transformations and $U, V$ be characteristic functions of them. Then, the characteristic function of the infinitesimal homogeneous contact transformation $[X, Y]$ is given by the Poisson bracket

$$
(U, V)=\phi^{\lambda \mu} \partial_{\lambda} U \partial_{\mu} V .
$$

PROOF. By virtue of (5. 6), we can easily verify that

$$
[X, Y]^{\lambda}=-\phi^{\lambda \mu} \partial_{\mu}\left(\phi^{\alpha \beta} \partial_{\alpha} U \partial_{\beta} V\right)
$$

which shows that our assertion is true.

THEOREM 5.4. The set $C$ of all coray functions of degree 1 over the cotangent bundle ${ }^{c} T\left(M^{n}\right)$ constitutes a Lie algebra with respect to the natural addition and the bracket operation (5. 9).

Proof. As we can easily see that

$$
\begin{aligned}
(U, V) & =-(V, U), \\
(U,(V+W)) & =(U, V)+(U, W)
\end{aligned}
$$

hold good, we only need to show that the Jacobi identity

$$
((U, V), W)+((V, W), U)+((W, U), V)=0
$$

holds good. However, as

$$
((U, V), W)=\phi^{\lambda \mu} \phi^{\rho \nu}\left(\partial_{\lambda} \partial_{\rho} U \partial_{\mu} V+\partial_{\lambda} U \partial_{\mu} \partial_{\rho} V\right) \partial_{\nu} W
$$


adding other two similar terms, we can easily see that (5.10) is true. Hence, the theorem is proved.

THEOREM 5.5. If we define the map

$$
h: C \rightarrow L
$$

$b y$

$$
U \rightarrow-\phi^{\lambda \mu} \partial_{\mu} U, \quad U \in C
$$

then $h$ is an isomorphism of $C$ onto $L$.

PROOF. First it is clear that $h$ is an homomorphism of $C$ onto $L$ if we regard them merely as additive groups. So, to prove that $h$ is a homomorphism of the Lie algebra $C$ onto the Lie algebra $L$, it is sufficient to show

$$
h(U, V)=[h U, h V] \text {. }
$$

However, the last equation can be written as

$$
-\phi^{\lambda \mu} \partial_{\mu}\left(\phi^{\alpha \beta} \partial_{\alpha} U \partial_{\beta} V\right)=\left[\phi^{\lambda \alpha} \partial_{\alpha} U, \phi^{\lambda \beta} \partial_{\beta} V\right]
$$

and its equality is already verified in the proof of Theorem 5.2. So, $h$ is a homomorphism.

Now, the kernel of $h$ is equal to zero, because if

$$
\phi^{\lambda \mu} \partial_{\mu} U=0
$$

we have $U=$ const. and hence $U$ has to be equal to zero.

COROLLARY 1. If we have $k$ parametric Lie group $G_{k}$ of homogeneous contact transformations of a differentiable manifold $M^{n}$, we denote $k$ infinitesimal homogeneous contact transformations which generate $G_{k}$ by $X_{p}(p=1$ $\cdots, k)$ and their characteristic functions by $U_{p}$. Then, $U_{p}$ 's are linearly independent with respect to constant coefficients and satisfy the relation

$$
\left(U_{p}, U_{q}\right)=c_{p_{q}}{ }^{r} U_{r}(p, q, r=1, \cdots, k)
$$

where $c_{p_{1}}^{r}$ are constant.

N.B. $\left(U_{p}, U_{q}\right)=0$ is the necessary and sufficient condition for the commutativity of the group generated by $U_{1}, \cdots, U_{k}$.

COROLlARY 2. The Lie algebra $L$ of all infinitesimal homogeneous contact transformations of $M^{n}$ is infinite dimensional.

Proof. As the Lie algebra $L$ and $C$ are isomorphic and $\operatorname{dim} C$ is infinity, so $\operatorname{dim} L$ is equal to infinity.

Now, we shall prove the

THEOREM 5.6. If a differentiable manifold $M^{n}$ is compact, then every 
infinitesimal homogeneous contact transformation $X$ generates a global one parameter group of global homogeneous contact transformations of $M^{n}$.

ProOF. We take a point $z_{0} \in{ }^{c} T\left(M^{n}\right)$ and a coordinate neighborhood $U\left(x^{i}\right)$ of $\pi z_{0}$. In $\pi^{-1}(U)$, we consider the set of differential equations of the type

$$
\frac{d x^{\lambda}}{d t}=X^{\lambda}
$$

Then, by virtue of the classical existence theorem on ordinary differential equations we can find a neighborhood $V\left(z_{0}\right)$ in $\pi^{-1}(U)$ and a positive constant $\varepsilon\left(z_{0}\right)$ so that

(a) (5.14) admits a solution

$$
x^{\lambda}=f_{t}^{\lambda}\left(z_{*}\right) \quad|t|<\varepsilon\left(z_{0}\right)
$$

with the initial condition $f_{0}\left(z_{*}\right)=z_{*}$ for every point $z_{*}$ of $V\left(z_{0}\right)$ and

(b) $f_{t}$ for every $|t|<\varepsilon\left(z_{0}\right)$ is a diffeomorphism of $V\left(z_{0}\right)$ onto its image under $f_{t}$ and

(c) if $t, t^{\prime}$ and $t+t^{\prime}$ belong to the interval $\left(-\varepsilon\left(z_{0}\right),+\varepsilon\left(z_{0}\right)\right)$, then

$$
f_{t} \circ f_{t^{\prime}}=f_{t+t^{\prime}}
$$

holds good.

The number $\varepsilon\left(z_{0}\right)$ generally depends upon the choice of $z_{0}$. On account of this fact, an infinitesimal homogeneous contact transformation generally may not generate a group of global homogeneous contact transformations. However, it is known that if we can choose $\varepsilon\left(z_{0}\right)$ so that it does not depend upon the choice of $z_{0}$, then the infinitesimal homogeneous contact transformation generates a global one parameter group $G_{1}$ of global homogeneous contact transformations.

Now, we define a transformation $T_{c}$ by

$$
T_{c}(x, p)=(x, c p)
$$

where $c$ is a positive constant. $T_{c}$ for $0<c<\infty$ is the one parametric multiplicative group generated by $\xi^{\lambda}$. So by (5. 7), $X$ is invariant under $T_{c}$ and hence we may take $\varepsilon\left(z_{0}\right)$ as $\varepsilon\left(T_{c} z_{0}\right)$. Accordingly $\varepsilon\left(z_{0}\right)$ depends only upon the coray on which $z_{0}$ lies.

Therefore, it is clear that $\varepsilon\left(z_{0}\right), z_{0} \in{ }^{c} T\left(M^{n}\right)$ has a positive greatest lower bound if $M^{n}$ is compact. Hence, our theorem is proved.

Now, let us introduce a positive definite Riemannian metric $g$ over $M^{n}$. Then, the set of all unit covariant vectors of $M^{n}$ constitutes a submanifold of ${ }^{c} T\left(M^{n}\right)$, which we denote by ${ }^{c} T_{1}\left(M^{n}\right)$. Any differentiable function $W\left(x, p_{1}\right)$ defined over $T_{1}\left(M^{n}\right)$ such that $W\left(x,-p_{1}\right)=W\left(x, p_{1}\right)$, where $p_{1} \in{ }^{c} T\left(M^{n}\right)$, can be easily extended to a coray function of degree 1 over ${ }^{c} T\left(M^{n}\right)$.

Hence, by virtue of the last theorem, we get the following 
THEOREM 5.7. If $M^{n}$ is a compact differentiable manifold, then there always exist homogeneous contact transformations.

6. Contact dstribution of the first kind. The tangent $n$-space to the fibre at a point $z=(x, p)$ of ${ }^{c} T\left(M^{n}\right)$ is called to be the vertical space at $z$. We consider an $n$-space which is disjoint and complementary to the vertical space at $z$ and call it as a transversal space to the vertical space at $z$.

In a coordinate neighborhood $\pi^{-1}(U)$ with coordinates $\left(x^{i}, p_{i}\right)$, we put

$$
\partial_{i}=\frac{\partial}{\partial x^{i}}, \partial^{i}=\frac{\partial}{\partial p_{i}} .
$$

If $\lambda_{j}{ }^{i} \partial_{i}+\mu_{j i} \partial^{i}$ are $n$ vectors which span the transversal $n$-space, then their natural projections $\lambda_{j}{ }_{j} \partial_{i}$ have to be linearly independent, so we have $\left|\lambda_{j}{ }_{j}\right| \neq 0$. Therefore, we may assume that $n$-vectors which span the transversal $n$-space have the form

$$
e_{i}=\partial_{i}+\Gamma_{i j} \partial^{j}
$$

We assume that

$$
\Gamma_{i j}=\Gamma_{j i},
$$

then we can see that it is independent upon the choice of local coordinates. To show it, let

$$
\bar{x}^{a}=\bar{x}^{a}(x), \bar{p}_{a}=p_{i} \bar{\partial}_{a} x^{i}
$$

be a coordinate transformation of local coordinates and its extension, then we can easily verify that

$$
\bar{\partial}_{a}+\bar{\Gamma}_{a b} \bar{\partial}^{b}=\bar{\partial}_{a} x^{i}\left(\partial_{i}+\Gamma_{i j} \partial^{j}\right),
$$

where we have put

$$
\bar{\Gamma}_{a b}=p_{k} \bar{\partial}_{a} \bar{\partial}_{b} x^{k}+\bar{\partial}_{a} x^{i} \bar{\partial}_{b} x^{j} \Gamma_{i j} .
$$

Therefore, we have $\bar{\Gamma}_{a b}=\bar{\Gamma}_{b a}$, which shows that our assertion is true.

Hereafter we consider a distribution $D_{1}$ of transversal $n$-spaces such that the symmetry condition (6. 3) is satisfied at every point of ${ }^{c} T\left(M^{n}\right)$. We call such distribution as contact distributions of the first kind and each of the set of $n$-vectors $e_{i}$ as contact frame of the first kind belonging to it and corresponding to the coordinate neighborhood in consideration. We say that $\Gamma_{i j}$ 's are parameters of the contact frame.

EXAMPLE. Let $\Gamma_{i j}^{k}$ be a symmetric affine connection defined over $M^{n}$. Then, we can easily verify that 


$$
\Gamma_{i j}=\Gamma_{i j}^{k} p_{k}
$$

defines a contact distribution of the first kind.

Now, we consider the vectors $e_{i}$ as operators in the sense

$$
e_{i} f=\partial_{i} f+\Gamma_{i j} \partial^{j} f
$$

for any function $f$ over ${ }^{c} T\left(M^{n}\right)$ and define a quantity defined by

$$
R_{i j k}=e_{k} \Gamma_{i j}-e_{j} \Gamma_{i k}
$$

Then we get the following

THEOREM 6.1. The contact distribution $D_{1}$ of the first kind is completely integrable if and only if

$$
R_{i j k}=0 .
$$

Proof. $n$-planes of $D_{1}$ are spanned by vectors $e_{i}$. As $e_{j}$ for fixed $j$ has components $\left(\delta_{j}{ }^{i}, \Gamma_{j i}\right)$ with respect to natural frames, we can easily see that

$$
\begin{aligned}
& {\left[e_{j}, e_{k}\right]^{i}=0,} \\
& {\left[e_{j}, e_{k}\right]^{n+i}=-R_{i j k} .}
\end{aligned}
$$

So, $\left[e_{j}, e_{k}\right]$ is a linear combination of $e_{r}$ if and only if $R_{i j k}=0$. Hence, the theorem follows.

COROllaRY. If the contact distribution $D_{1}$ of the first kind is the one which is associated to a symmetric affine connection by (6. 5). then $D_{1}$ is completely integrable if and only if the affine connection is flat.

PROOF. We can easily verify that

$$
R_{i j k}=R_{i j k}^{h} p_{h},
$$

where $R^{h}{ }_{i j k}$ 's are components of the curvature tensor of the affine connection. As $p_{h}$ 's are arbitrary, we have $R^{h}{ }_{i j k}=0$ if $R_{i j k}=0$. Hence, the theorem is proved.

THEOREM 6.2. The contact distribution $D_{1}$ of the first kind is invariant under the transformation $T_{c}$ if and only if $\Gamma_{i j}(x, p)$ 's are coray functions of degree 1.

ProOF. As the $n$-space of the contact distribution of the first kind at $z=(x, p)$ is spanned by $n$-vectors with components $\left(\delta_{a}^{i}, \Gamma_{a i}(x, p)\right)(a=1, \cdots, n)$ we can easily see that it is defined by equations $\boldsymbol{\omega}_{n+i}=0$, where we have put

$$
\omega_{n+i}=d p_{i}-\Gamma_{i j}(x, p) d x^{j} .
$$

The equations $\omega_{n+i}=0$ at ' $z=T_{c} z$ are satisfied by vectors of the $n$-space which is the image of the $n$-space of $D_{1}$ at $z$ under $T_{c}$ if and only if 
$\Gamma_{i j}(x, p)$ 's are coray functions of degree 1 . Hence, the theorem is proved.

Let $U_{\lambda}(\lambda \in \Lambda)$ be an open covering of $M^{n}$ and $f$ be a homogeneous contact transformation of $M^{n}$. Suppose $f \circ \pi^{-1}\left(U_{\lambda}\right) \cap \pi^{-1}\left(U_{\mu}\right)$ is not empty, then the restriction map

$$
f: \pi^{-1}\left(U_{\lambda}\right) \cap f^{-1} \circ \pi^{-1}\left(U_{\mu}\right) \rightarrow f \circ \pi^{-1}\left(U_{\lambda}\right) \cap \pi^{-1}\left(U_{\mu}\right)
$$

can be expressed by

$$
\bar{x}^{a}=\bar{x}^{a}(x, p), \bar{p}_{a}=\bar{p}_{a}(x, p),
$$

where $\left(x^{i}, p_{i}\right)$ are coordinates in $\pi^{-1}\left(U_{\lambda}\right)$ and $\left(\bar{x}^{a}, \bar{p}_{a}\right)$ are coordinates in $\pi^{-1}\left(U_{\mu}\right)$.

Now, in order to get good insight of the complicated calculations, we introduce matrix notation

$$
\begin{cases}A=\left(\partial_{i} \bar{x}^{a}\right), & B=\left(\partial^{i} \bar{x}^{a}\right), \\ C=\left(\partial_{i} \bar{p}_{a}\right), & D=\left(\partial^{i} \bar{p}_{a}\right)\end{cases}
$$

and

$$
\begin{cases}\bar{A}=\left(\bar{\partial}_{a} x^{i}\right), & \bar{B}=\left(\bar{\partial}^{a} x^{i}\right), \\ \bar{C}=\left(\bar{\partial}_{a} p_{i}\right), & \bar{D}=\left(\bar{\partial}^{a} p_{i}\right) .\end{cases}
$$

Then, by virtue of (2.22) and (2.18), we have

$$
\left\{\begin{aligned}
A & ={ }^{t} \bar{D}, & B & =-{ }^{t} \bar{B}, \\
C & =-{ }^{t} \bar{C}, & D & ={ }^{t} \bar{A}
\end{aligned}\right.
$$

and

$$
\left\{\begin{array}{l}
B^{t} A=A^{t} B, \quad D^{t} C=C^{t} D, \\
B^{t} C-A^{t} D=-E,
\end{array}\right.
$$

where $t$ 's on the left shoulders of matrices mean their transposes and $E$ is the unit matrix. It is evident that we have also the identities

$$
\left\{\begin{array}{rlrl}
A \bar{A}+B \bar{C}=E, & & A \bar{B}+B \bar{D}=0, \\
C \bar{A}+D \bar{C}=0, & C \bar{B}+D \bar{D}=E .
\end{array}\right.
$$

Now, if

$$
|A+B \Gamma| \neq 0
$$

at a point $z=(x, p)$ or $\pi^{-1}\left(U_{\lambda}\right) \cap f^{-1} \circ \pi^{-1}\left(U_{\mu}\right)$, we say that $f$ is regular at $z$ with respect to the contact distribution $D_{1}$. And if $f$ is regular at every point of $T\left(M^{n}\right)$, we say that $f$ is regular with respect to $D_{1}$. The independence of the notion of regularity upon coordinate neighborhood comes from the following

THEOREM 6.3. If a homogeneous contact transformation $f$ of $M^{n}$ is 
regular with respect to a contact distribution of the first kind $D_{1}$ at a point $z=(x, p)$ of ${ }^{c} T\left(M^{n}\right)$, then the image of the $n$-space of $D_{1}$ at the point $z$ is also transversal to the vertical space at the point $f(z)$. The converse is also true.

PROOF. The transversal $n$-space of $D_{1}$ at the point $z$ is spanned by $n$ vectors $\boldsymbol{e}_{i}$.

If we fix $i$, the components of the vector $e_{i}$ with respect to the natural frame $\left(\partial_{j}, \partial^{j}\right)$ are $\left(\delta_{i}^{j}, \Gamma_{i j}\right)$. So, the components of the image of the vector under the transformation $f$ are easily seen to be given by the $i$-th columns of the set of matrices $(A+B \Gamma, C+D \Gamma)$. Therefore, the $\pi$-image of the vector in consideration has as its components the $i$-th column of $A+B \Gamma$. Hence, the condition (6.16) is equivalent to the fact that the $\pi \circ f$ image of the transversal $n$-space at the point $z$ spanned by $n$ vectors $e_{i} \quad(i=1, \cdots, n)$ coincides with the tangent space of $M^{n}$ at the point $\pi \circ f(z)$. Hence, the condition (6.16) is also equivalent to the fact that the $f$-image of the $n$-space of $D_{1}$ at the point $z$ is again a transversal $n$-space at $f(z)$.

It is evident that the converse is also true.

Now, assuming that $D_{1}$ is a contact distribution of the first kind and $f$ is a homogeneous contact transformation we consider equations

for unknowns $\bar{\Gamma}_{a b}$.

$$
\bar{\Gamma}(A+B \Gamma)=C+D \Gamma^{1)}
$$

LEMMA 6.1. In order that (6.17) admits a set of solutions $\overline{\mathbf{T}}_{f b}$ at a point $f(z) \in f \circ \pi^{-1}\left(U_{\lambda}\right) \cap \pi^{-1}\left(U_{\mu}\right)$, it is necessary and sufficient that $f$ is regular with respect to $D_{1}$ at the point $z \in \pi^{-1}\left(U_{\lambda}\right) \cap f^{-1} \circ \pi^{-1}\left(U_{\mu}\right)$.

ProOF. Sufficiency is evident.

Necessity. By virtue of (2.22), we can write (6.17) as

So we have

$$
\bar{\Gamma}\left({ }^{t} \bar{D}-{ }^{t} \bar{B} \Gamma\right)=-{ }^{t} \bar{C}+{ }^{t} \bar{A} \Gamma \text {. }
$$

$$
\Gamma\left(\bar{A}+\bar{B}^{t} \underset{f}{\bar{\Gamma}}\right)=\bar{C}+\bar{D}_{f}^{t} \bar{\Gamma}
$$

1) If we consider homogeneous contact transformation (2.13) as a coordinate transformation and introduce an object $\Gamma$ which is transformed by

$$
\overline{\boldsymbol{\Gamma}}(A+B \mathbf{r})=C+\bar{D} \mathbf{r}
$$

under such coordinate transformation, then we have

$$
d p-\overline{\mathbf{\Gamma}} d x=(D-\overline{\mathbf{\Gamma}} B)(d p-\mathbf{\Gamma} d x) .
$$

So we may define a parallel displacement of $p_{i}$ which is invariant under homogeneous contact transformations by $d p-\Gamma d x=0$. The object $\Gamma$ and the equation $\left(^{*}\right)$ were first introduced by T. Hosokawa [7] in this way. The word contact frame was first used by L. P. Eisenhart [6] without explicit mention of the vectors $\partial_{i}+\Gamma_{i} y^{j}$. 
which is similar to (6.17).

On the other hand, we have

$$
\begin{aligned}
& (A+B \Gamma)\left(\bar{A}+\bar{B}^{\bar{T}} \bar{T}\right) \\
& =A \bar{A}+{ }^{f} \bar{B}^{t}{ }_{f}^{\bar{\Gamma}}+B \Gamma\left(\bar{A}+\bar{B}_{f}{ }_{f}^{\bar{T}}\right) .
\end{aligned}
$$

The right hand side is easily transformed to

$$
E-B\left\{\bar{C}+\bar{D}_{f}^{t} \bar{\Gamma}_{f}-\Gamma\left(\bar{A}+\bar{B}_{f}^{t} \bar{\Gamma}\right)\right\} .
$$

Therefore, by (6.18), we get

$$
(A+B \Gamma)\left(\bar{A}+\bar{B}^{t} \bar{\Gamma}\right)=E .
$$

Hence, we have (6.16) which shows that $f$ is regular at the point $z=(x, p)$.

\section{symmetric.}

LEMMA 6.2. If (6.17) admits a set of solutions $\bar{\Gamma}_{f}$, then $\bar{\Gamma}_{a b}$ 's are

Proof. We multiply $\bar{A}+\bar{B}^{t} \bar{\Gamma}$ to both sides of (6.17). Then, we see first that the left hand side reduces to $\bar{\Gamma}_{f}$ by virtue of (6.19). Secondly, the right hand side is transformed to

$$
\begin{aligned}
& (C+D \Gamma)\left(\bar{A}+\bar{B}^{t} \bar{\Gamma}\right) \\
& =(C+D \bar{\Gamma}) \bar{A}+C \bar{B}_{\mathcal{J}}^{t} \bar{\Gamma}+D \Gamma \bar{B}^{t} \bar{\Gamma} .
\end{aligned}
$$

Putting (6.18) into the last term of the right hand side of the last eqation, we see that the right hand side reduces to $\bar{\Gamma}$. Hence, replacing $c$ by $b$, we get

which is to be proved.

$$
\bar{\Gamma}_{f a b}=\bar{\Gamma}_{f}^{f}
$$

THEOREM 6.4. If a homogeneous contact transformation $f$ of $M^{n}$ is regular at a point $z$ of ${ }^{c} T\left(M^{n}\right)$ with respect to a contact distribution $D_{1}$ of the first kind determined by $\Gamma$, then the image of the n-space of $D_{1}$ at $z$ under $f$ is the $n$-space determined by $\bar{\Gamma}_{f}$ at $\bar{z}=f(z)$

PROOF. As the vector $e_{i}$ for fixed $i$ has components $\left(\delta_{i}^{j}, \Gamma_{i j}\right)$ with respect to the natural frame $\left(\partial_{j}, \partial^{j}\right)$, we can easily see that the image of this vector has as its components the $i$-th columns of the set of matrices $(A+B \Gamma, C+D \Gamma)$ with respect to the natrual frame $\left(\bar{\partial}_{a}, \bar{\partial}^{a}\right)$. We consider $n$ such vectors, and take a linear combinations of these vectors by multiplying $\bar{A}+\bar{B}^{t}{ }_{f}$, then we get $\left(\delta_{b}^{a}, \bar{\Gamma}_{a b}\right)$, i.e. we have $n$ vectors

$$
\bar{e}_{b}=\bar{\partial}_{b}+\bar{\Gamma}_{b c} \bar{\partial}^{c} .
$$

Hence, the image of the transversal $n$-space determined by $\Gamma$ at $z$ is the transversal $n$-space determined by $\underset{f}{\bar{\Gamma}}$ at $f(z)$. 
From the proof of Theorem 6.3, we can see that

$$
\bar{e}_{b}=\left(\bar{\partial}_{b} x^{i}+\bar{\partial}^{c} x^{i} \bar{\Gamma}_{b c}\right) f\left(e_{i}\right),
$$

where $f\left(e_{i}\right)$ is the vector which is the image of $e_{i}$ under $f$.

From Theorems 6.3 and 6.4 we get the following

THEOREM 6.5. Let $D_{1}$ be a contact distribution of the first kind of ${ }^{c} T\left(M^{n}\right)$ determined by $\Gamma$ and $f$ be a homogeneous contact transformation of $M^{n}$. If $f$ is regular at every point of ${ }^{c} T\left(M^{n}\right)$ with respect to $D_{1}$, then $f$ induces a new contact distribution $f D_{1}$ of the first kind in ${ }^{c} T\left(M^{n}\right)$.

Of course, the $n$-plane of $f D_{1}$ at $f(z)$ is spanned by $\bar{e}_{a}$. We denote the vectors of $f D_{1}$ at $z$ by $\partial_{i}+\Gamma_{j} \partial^{j}$.

If a homogeneous contact transformation $f$ satisfies the relation

$$
\Gamma=\Gamma
$$

then we say that the contact distribution $D_{1}$ determined by $\Gamma$ is invariant under $f$. We shall study homogeneous contact transformations which leave $\Gamma$ invariant.

As an example we shall prove the following

THEOREM 6.6. Suppose $D_{1}$ is the contact distribution of the first kind associated with a symmetric affine connection $\Gamma$ of $M^{n}$. (i) The extention of every affine transformation of $M^{n}$ leaves $D_{1}$ invariant. (ii) If the extended group of a Lie group $G$ of diffeomorphisms of $M^{n}$ leaves $D_{1}$ invariant, $G$ is a group of affine transformations.

PROOF. Let us take the local expression of $f$ as in (6.10). In order that the contact distribution of the first kind $D_{1}$ is invariant under $f$ it is necessary and sufficient that

$$
\Gamma_{a b}(\bar{x}, \bar{p})\left(\partial_{i} \bar{x}^{b}+\partial^{j} \bar{x}^{j} \Gamma_{j i}\right)=\partial_{i} \overline{p_{a}}+\partial^{j} \bar{p}_{a} \Gamma_{j i} .
$$

(i) Suppose $f$ be an extension of an affine transformation of $M^{n}$ with a symmetric affine connection $\Gamma$. Then, we have

$$
\bar{x}^{a}=\bar{x}^{a}(x), \bar{p}_{a}=p_{i} \bar{\partial}_{a} x^{i}
$$

and

$$
\Gamma_{a b}^{c}(\bar{x})=\partial_{k} \bar{x}^{n}\left(\bar{\partial}_{a} \bar{\partial}_{b} x^{k}+\Gamma_{? j}^{k} \bar{\partial}_{a} x^{i} \bar{\partial}_{b} x^{j}\right)
$$

Noticing (6. 5) and making use of (6.25) ${ }_{2}$ and (6.26) we get

$$
\Gamma_{a b}(\bar{x}, \bar{p})=\bar{\partial}_{a} \bar{\partial}_{b} x^{k} p_{k}+\Gamma_{i j}(x, p) \bar{\partial}_{a} x^{i} \bar{\partial}_{b} x^{j}
$$

Comparing the last equation with (6.23) we see that $D_{1}$ is invariant under $f$.

(ii) We consider $U_{\mu}$ coincides with $U_{\lambda}$ and take an infinitesimal homogeneous contact transformation defined by 


$$
\bar{x}^{i}=x^{i}+\partial^{i} U \delta t, \overline{p_{i}}=p_{i}-\partial_{i} U \delta t,
$$

where $U$ is a coray function of degree 1 over ${ }^{c} T\left(M^{n}\right)$. From (6.27), we can easily see that the transformation (6.28) leaves $D_{1}$ invariant if and only if the equation

$$
\partial_{k} \Gamma_{i a} \partial^{k} U-\partial^{k} \Gamma_{i a} \partial_{k} U+\Gamma_{i j} e_{a} \partial^{j} U+e_{a} \partial_{i} U=0 .
$$

Now, we consider (6.28) which is the extension of an infinitesimal diffeomorphism defined by a vector field $X^{i}$ of $M^{n}$.

Putting (6. 5) into (6.29), we have

$$
X^{h}{ }_{i a}+R_{i a b}^{h} X^{k}=0 \text {, i.e. } \mathcal{L}_{(}(X) \Gamma_{i a}^{h}=0 .
$$

So, $X^{i}$ is an infinitesimal affine transformation. If we take vector fields which generate the given Lie group $G$ instead of $X^{i}$, we can see that $G$ is a Lie group of affine transformations of $M^{n}$.

7. Contact distribution of the second kind. We consider another $n$ dimensional distribution $D_{2}$ such that the $n$-space of $D_{2}$ is disjoint and complementary to the $n$-space of the contact distribution of the first kind $D_{1}$ at every point of ${ }^{c} T\left(M^{n}\right)$. As the bases of $n$-spaces of $D_{2}$, we may take $n$ vectors of the form $\lambda_{i}^{j} e_{j}+\mu_{i j} \partial^{j}$. As the $n$-space of $D_{2}$ is disjoint and complementary to the $n$-space of $D_{1}$ at every point of ${ }^{c} T\left(M^{n}\right)$, we have $\left|\mu_{j}^{i}\right| \neq 0$. So we may assume that the bases are given by $n$ vectors of the form

$$
e^{i}=\partial^{i}-\Pi^{i j}\left(\partial_{j}+\Gamma_{j k} \partial^{k}\right) .
$$

Here, we assume that

$$
\Pi^{i j}=\Pi^{j i}
$$

The assumption (7. 1) is independent upon the choice of local coordinates. For, if (6. 4) is a coordinate transformation and it extension, then we have

$$
\begin{aligned}
& \partial^{i}-\Pi^{i j}\left(\partial_{j}+\Gamma_{j k} \partial^{k}\right) \\
& \quad=\partial^{i} \bar{p}_{a} \bar{\partial}_{a}-\Pi^{i j} \partial_{j} \bar{x}^{b}\left(\overline{\partial_{b}}+\bar{\Gamma}_{b c} \bar{\partial}^{c}\right)
\end{aligned}
$$

by virtue of an analogous argument for $\Gamma_{i j}$ in $\$ 6$. The last equation is easily transformed to $\bar{\partial}_{a} x^{i}\left\{\bar{\partial}^{a}-\bar{\Pi}^{a b}\left(\bar{\partial}_{b}+\bar{\Gamma}_{b c} \bar{\partial}^{c}\right)\right\}$, where we have put

$$
\bar{\Pi}^{a b}=\Pi^{i j} \partial_{i} \bar{x}^{a} \partial_{j} \bar{x}^{b} .
$$

Hence, $D_{2}$ is spanned by $e^{a}=\bar{\partial}^{a}-\bar{\Pi}^{a b}\left(\bar{\partial}_{b}+\bar{\Gamma}_{b c} \bar{\partial}^{c}\right)$ and $\bar{\Pi}^{a b}$ s are symmetric.

We call such distribution $D_{2}$ as a contact distribution of the second kind assocciated to the contact distribution of the first kind $D_{1}$. The set of $n$ vectors $e^{i}$ is called a contact frame of the second kind. We say that $\Pi^{j i}$ 's are parameters of the contact frame.

EXAMPLE 1. The distribution determined by the set of all vertical $n$-spaces 
of ${ }^{c} T\left(M^{n}\right)$.

EXAMPLE 2. We endow a positive definite Riemannian metric $g$ to $M^{n}$ and define

$$
\Gamma_{i j}=\left\{\begin{array}{l}
k \\
i j
\end{array} \mid p_{k}, \quad \Pi^{i j}=g^{i j} / \sqrt{g^{n k} p_{h} p_{k}}\right.
$$

at every coordinate neighborhood $\pi^{-1}(U)$ of ${ }^{c} T\left(M^{n}\right)$, where $U$ is a coordinate neighborhood of $M^{n}$ and $g^{i j},\left\{\begin{array}{l}k \\ i j\end{array}\right\}$ are the fundamental tensors and the Christoffel's symbols of the Riemannian manifold $M^{n}$. Then, $\Gamma_{i j}$ and $\Pi^{i j}$ determine contact distributions of the first and second kind.

THEOREM 7.1. Suppose the contact distribution $D_{1}$ of the first kind is invariant under the transformation $T_{c}$. Then, the contact distribution $D_{2}$ of the second kind is invariant under $T_{c}$, if and only if $\Pi^{i j}(x, p)$ 's are coray functions of degree -1 .

PROof. As the $n$-space of $D_{2}$ at a point $z=(x, p)$ is spanned by $n$ vectors $e^{a}$ with components $\left(-\Pi^{a i}, \delta_{i}^{a}-\Pi^{a b} \Gamma_{b i}\right)$, we can easily verify that it is defined also by $n$ equations $\omega_{i}=0$, where we have put

$$
\omega_{i}=d x^{i}+\Pi^{i j}(x, p)\left\{d p_{j}-\Gamma_{j k}(x, p) d x^{k}\right\} .
$$

The equations of the type $\omega_{i}=0$ at $T_{c} z$ are satisfied by vectors of the $n$-space which is the image of the $n$-space of $D_{1}$ at $z$ under $T_{c}$ if and only if $\Pi^{i j}$ s are coray functions of degree -1 as $\Gamma_{i j}$ 's are coray functions of degere +1 by assumption. Hence, our theorem is proved.

Now, we consider the vectors $e^{i}$ as operators in the sense

$$
e^{i} f=\partial^{i} f-\Pi^{i j}\left(\partial_{j} f+\Gamma_{j k} \partial^{k} f\right)
$$

and put

$$
R^{i j k}=e^{k} \Pi^{i j}-e^{j} \Pi^{i k}+\Pi^{i h}\left(\Pi^{a j} e^{k} \Gamma_{a h}-\Pi^{a k} e^{j} \Gamma_{a h}\right) .
$$

Then, we get the following

THEOREM 7.2. The contact distribution of the second kind $D_{2}$ is completely integrable if and only if there exist the relations

$$
R^{i j k}=0
$$

PROOF. $n$-spaces of $D_{2}$ are spanned by $n$ vectors $e^{i}$ defined by (7. 1). As $e^{a}$ for fixed $a$ has components $\left(-\Pi^{i a}, \delta_{i}^{a}-\Pi^{a b} \Gamma_{b i}\right)$ with respect to natural frames, we can easily see that

$$
\begin{aligned}
& {\left[e^{j}, e^{k}\right]^{i}=e^{k} \Pi^{i j}-e^{j} \Pi^{i k},} \\
& {\left[e^{j}, e^{k}\right]^{n+i}=e^{k}\left(\Gamma_{i a} \Pi^{a j}\right)-e^{j}\left(\Gamma_{i a} \Pi^{a k}\right) .}
\end{aligned}
$$


On the other hand, by the theory of distributions the distribution $D_{2}$ is completely integrable if and only if there exist functions $\lambda^{j k}{ }_{h}$ such that

$$
\left[e^{j}, e^{k}\right]=\lambda^{j k}{ }_{h} e^{h},
$$

i.e.

$$
\begin{aligned}
& e^{k} \Pi^{i j}-e^{j} \Pi^{i k}=-\lambda_{h}^{j k} \Pi^{h i}, \\
& e^{k}\left(\Gamma_{i a} \Pi^{a j}\right)-e^{j}\left(\Gamma_{i a} \Pi^{a k}\right)=\lambda_{h}^{j k}\left(\delta_{i}^{h}-\Pi^{h l} \Gamma_{l i}\right) .
\end{aligned}
$$

Eliminating $\lambda^{j k}{ }_{h}$ from the last two equations we see that our assertion is true.

COROLlaRY. If the distribution of the second kind $D_{2}$ can be transformed to the distribution determined by vertical $n$-spaces by a homogeneous contact transformation $f$, then (7. 7) holds good.

ProOF. The distribution determined by the vertical $n$-spaces are completely integrable. So, its inverse image $D_{2}$ by $f^{-1}$ is also completely integrable. Hence, by virtue of Theorem 7.3, we see that our assertion is true.

Let $f$ be a homogeneous contact transformation which is regular with respect to the contact distribution $D_{1}$. We consider now the equations

$$
\bar{\Pi}_{f}\left({ }^{t} \bar{A}+\bar{\Gamma}_{f}{ }^{t} \bar{B}\right)=(A+B \Gamma) \Pi-B .^{2)}
$$

Then, $\bar{\Pi}_{f}^{a b}$ s are defined in $f \circ \pi^{-1}\left(U_{\lambda}\right) \cap \pi^{-1}\left(U_{\mu}\right)$ uniquely, as

by virtue of (6.19).

$$
\left|\bar{A}+\bar{B}{ }^{t} \bar{\Gamma}\right| \neq 0
$$

LEMMA 7.1. $\Pi_{f}^{a b}$ 's defined by (7.8) are symmetric with respect to a and $b$.

Proof. We multiply $A+B \Gamma$ to both sides of (7.8) and contract with respect to $i$. Then the left hand side reduces to $\bar{\Pi}_{f}$ by virtue of (6.19), the right hand side reduces to

$$
(A+B \Gamma) \Pi\left({ }^{t} A+\Gamma^{t} B\right)-B{ }^{t} A-B \Gamma{ }^{t} B .
$$

It is evident that the first term and the third term of the last formula are symmetric. The second term is also symmetric by virtue of $(6.14)_{1}$. So the right hand side is symmetric. Hence we can see that

$$
\bar{\Pi}^{a b}=\bar{\Pi}^{b a} .
$$

2) Considering the homogeneous contact transformation (2.13) as a coordinate transformation, Y. Muto [8] and T. C. Doyle [3] independently introduced the object $\Pi$ which is transformed by an equation of the form (7.8). 
The geometric meaning of $\Pi_{J}^{a b}$ is given by the following

THEOREM 7.3. If a homogeneous contact transformation $f$ of $M^{n}$ is regular with respect to the contact distribution $D_{1}$ determined by $\Gamma$, then the image of the n-space spanned by $e^{i}$ under $f$ is the $n$-space spanned by $\bar{\partial}^{a}-\bar{\Pi}^{a b}\left(\overline{\partial_{b}}+\bar{\Gamma}_{b} \bar{\partial}^{c}\right)$.

PROOF. As the vector $e^{j}$ for a fixed $j$ has components $\left(-\Pi^{i j}, \delta_{j}^{i}-\Gamma_{i k} \Pi^{k j}\right)$ with respect to the natural frame $\left(\partial_{i}, \partial^{i}\right)$, the image of the vector under the homogeneous contact transformation $f$ has as its components the $j$-th columns of the set of matrices

$$
\{B-(A+B \Gamma) \Pi, D-(C+D \Gamma) \Pi\}
$$

with respect to the natural frame $\left(\bar{\partial}_{a}, \bar{\partial}^{a}\right)$. By virtue of (7.18), this is transformed to

$$
\left\{-\bar{\Pi}_{f}\left({ }^{t} \bar{A}+\bar{\Gamma}_{f}{ }^{t} \bar{B}\right), D-(C+D \Gamma) \Pi\right\} .
$$

Now, we take linear combinations of these $n$ vectors by multiplying ${ }^{t}(A+B \Gamma)$ and summing up for $j$, we get by (6.17)

$$
\left\{-\bar{\Pi}_{\boldsymbol{f}}, D^{t}(A+B \Gamma)-(C+D \Gamma) \Pi^{t}(A+B \Gamma)\right\} .
$$

On the other hand we have

So, we have

$$
\begin{gathered}
\bar{\jmath}=(A+B \Gamma) \Pi^{t}(A+B \Gamma)-B^{t}(A+B \Gamma), \\
\bar{\Gamma}_{J}(A+B \Gamma)=C+D \Gamma .
\end{gathered}
$$

$$
\bar{\Gamma}_{f} \bar{\Gamma}=(C+D \Gamma) \Pi^{t}(A+B \Gamma)-\bar{\Gamma}_{f} B^{t}(A+B \Gamma) .
$$

By virtue of the last equation (7.11) can be written as

$$
\begin{aligned}
& \left\{-\prod_{f}, D^{t}(A+B \Gamma)-\bar{\Gamma} B^{t}(A+B \Gamma)-\bar{\Gamma}_{f} \Pi_{f}\right\} \\
& =\left\{-\prod_{f},(D-\bar{\Gamma} B)^{t}(A+B \Gamma)-\bar{\Gamma} \bar{\Pi}_{f}\right\} \\
& =\left\{-\prod_{f},{ }^{t}(\bar{A}+\bar{B} \bar{\Gamma})^{t}(A+B \Gamma)-\bar{\Gamma} \prod_{f}\right\} \\
& =\left\{-\bar{\Pi}_{f}, E-\bar{\Gamma} \overline{\Pi_{f}}\right\}
\end{aligned}
$$

by virtue of $(6.13)_{1,2}$ and (6.17). Therefore, the image of the $n$-space spanned by $e^{i}$ is spanned by

$$
\bar{e}^{a}=\bar{\partial}^{a}-\bar{\Pi}_{r}^{a b}\left(\bar{\partial}_{b}+\bar{\Gamma}_{f c} \bar{\partial}^{c}\right)
$$

From the proof of Theorem 7.1, we can see that 


$$
\bar{e}^{b}=\left(\partial_{j} \bar{x}^{b}+\Gamma_{h j} \partial^{h} \bar{x}^{b}\right) f\left(e^{j}\right),
$$

where $f\left(e^{j}\right)$ is the vector which is the image of $e^{j}$ under $f$.

From Theorems 6.5, 7.3 and Lemma 7.1 we get the following

THEOREM 7.4. Let $D_{1}$ and $D_{2}$ be contact distributions of the first and second kind of ${ }^{c} T\left(M^{n}\right)$ determined by $\Gamma$ and $\Pi$ and $f$ be a homogeneous contact transformation of $M^{n}$. If $f$ is regular at every point of $D_{1}$, then $f$ induces new contact distribution of the second kind $f D_{2}$ associated to $f D_{1}$.

$\bar{\Pi}_{f}^{a b}$ 's are parameters which define $f D_{2}$ in $f \circ \pi^{-1}\left(U_{\lambda}\right) \cap \pi^{-1}\left(U_{\mu}\right)$. We denote the parameters which define $f D_{2}$ generally by $\prod_{f}^{i j}$.

If a homogeneous contact transformation $f$ satisfies the relation

$$
\Pi=\Pi,
$$

then we say that the contact distribution $D_{2}$ determined by $\Gamma$ and $\Pi$ is invariant under $f$.

THEOREM 7.5. Suppose that we take the distribution determined by vertical spaces as contact distribution of the second kind. Then every homogeneous contact transformation $f$ which leaves this contact distribution invariant is an extension of a diffeomorphism of $M^{n}$.

Proof. Putting $\Pi=0, \bar{\Pi}=0$ into (7. 8) we get $\partial^{i} \bar{x}^{a}=0$. Noticing that the 1 -form $p_{i} d x^{i}$ is invariant under $f$, we can easily see that our contact transformation $f$ is an extension of a diffeomorphism of $M^{n}$.

THEOREM 7.6. Let $M^{n}$ be a Riemannian manifold and suppose that we take the contact distributions determined by (7. 4). Then the extension of an isometry of $M^{n}$ leaves both distributions $D_{1}$ and $D_{2}$ invariant. And if $G^{\prime}$ is the extended group of a Lie group $G$ of diffeomorphisms of $M^{n}$ such that every transformation of $G^{\prime}$ leaves $D_{1}$ and $D_{2}$ invariant, then $G$ is a group of isometries of $M^{n}$.

The proof is almost evident from that of Theorem 6.6 and the law of transformation (7. 3) of $\Pi^{i j}$ under an extension of a diffeomorphism of $M^{n}$.

THEOREM 7.7. If we denote the components of an arbitrary vector $X$ with respect to the natural frame by $\left(X^{i}, P_{i}\right)$, then its components with respect to the contact frame $\left(e_{i}, e^{i}\right)$ are given by

$$
\left\{\begin{array}{l}
\Lambda^{i}=X^{i}+\Pi^{i j} M_{j}=X^{i}+\Pi^{i j}\left(P_{j}-\Gamma_{j k} X^{k}\right), \\
M_{i}=P_{i}-\Gamma_{i j} X^{j} .
\end{array}\right.
$$

ProOF. We can easily verify that 


$$
\Lambda^{i} e_{i}+M_{i} e^{i}=X^{i} \partial_{i}+P_{i} \partial^{i},
$$

which shows that our assertion is true.

THEOREM 7.8. The projection tensors $T_{1}$ and $T_{2}$ of an arbitrary vector to the distributions $D_{1}$ and $D_{2}$ are given by

$$
\begin{gathered}
T=\left(\begin{array}{lc}
\delta_{j}^{i}-\Pi^{i k} \Gamma_{k j} & \Pi^{i j} \\
\Gamma_{i j}-\Gamma_{i k} \Pi^{k h} \Gamma_{h j} & \Gamma_{i k} \Pi^{k j}
\end{array}\right), \\
\underset{2}{T}=\left(\begin{array}{ll}
\Pi^{i k} \Gamma_{k j} & -\Pi^{i j} \\
-\Gamma_{i j}+\Gamma_{i k} \Pi^{k h} \Gamma_{h j} & \delta_{i j}-\Gamma_{i k} \Pi^{k j}
\end{array}\right)
\end{gathered}
$$

respectively with respect to natural frames.

PROOF. The projection of an arbitrary vector $X=\left(X^{i}, P_{i}\right)$ on $D_{1}$ is given by $\Lambda^{i} e_{i}$. The components of the last vector with respect to the natural frame are easily seen to be

$$
\left(\delta_{j}^{i}-\Pi^{i k} \Gamma_{k j}\right) X^{j}+\Pi^{i j} P_{j},\left(\Gamma_{i j}-\Gamma_{i k} \Pi^{k h} \Gamma_{h j}\right) X^{j}+\Gamma_{i k} \Pi^{k j} P_{j} .
$$

The components of $T_{1}$ are nothing but the coefficients of $X^{j}, P_{j}$ of the last vector. So, (7.16) is proved. The proof of (7.17) can be obtained in the same way.

Now we denote the projections of the image of a vector $X$ at $z$ by a homogeneous conact transformation $f$ on $f D_{1}$ and $f D_{2}$ by $\bar{\Lambda}^{a} \bar{e}_{a}$ and $\bar{M}_{a} \bar{e}^{a}$, then as $\Lambda^{i} e_{i}$ and $M_{i} e^{i}$ are transformed by $f$ to $\bar{\Lambda}^{a} \bar{e}_{a}$ and $\bar{M}_{a} \bar{e}^{a}$ respectively, we can easily see that

$$
\bar{\Lambda}=(A+B \Gamma) \Lambda, \bar{M}(A+B \Gamma)=M
$$

hold good by (6.22) and (7.13).

\section{BIBLIOGRAPHY}

[1] Davies, E. T., On the invariant theory of contact transformations, Math. Zeits., 57 (1953), 415-427.

[2] DAviES, E. T. and K. Yano, Contact tensor calculus, Ann. di Mat. , 37 (1954), 1-36.

[3] DOYLE, T. C. Tensor decomposition with applications to the contact and complex groups, Ann. of Math. 42 (1941), 698-721.

[4] Eisenhart, L. P., Contact transformations. Ann. of Math. (2) 30 (1929), 211-249.

[5] EISENHART, L. P., Continuous groups of transformations. Princeton Univ. Press, 1933.

[6] EISENHART, L. P. AND M.S. KNEBELMANN, Invariant theory of homogeneous contact transformations. Ann. of Math. 37 (1936), 747-765

[ 7] HosokAwA, T., Connections in the manifold admitting contact transformations. Journ. Fasc. Sci. Hokkaido Imp. Univ., 2 (1934), 169-176.

[8] MUTô, Y., On the connections in the manifold admitting homogeneous contact transformations. Proc. Phys. Math. Soc. Japan, 20 (1938), 451-457.

[9] Mutô, Y. AND K. YANO, Sur les transformations de contact et les espaces de Finsler, Tôhoku Math. Journ., 45 (1939), 293-307. 
[10] YaNo, K., On the theory of linear connections in the manifold admitting contact transformations, Proc. Phys. Math. Soc. Japan, 17 (1935), 39-47.

TÔHOKU UNIVERSITY 